\title{
Improving Health of Veterans through Research Collaborations
}

\author{
Patricia M. Hayes, PhD \\ Women's Health Services, Patient Care Services, Department of Veterans Affairs, VA Central Office, Washington, DC, USA.
}

J Gen Intern Med 28(Suppl 2):S495-7

DOI: $10.1007 / \mathrm{s} 11606-013-2471-8$

(c) Society of General Internal Medicine 2013

$\mathrm{T}$ his issue of the Journal of General Internal Medicine provides a timely opportunity to highlight some of the research collaborations sponsored by our program and to review strategic priorities that promote new collaborations in areas of greatest need for research. The mission of the Veterans Health Administration (VA) office of Women's Health Services (WHS) is really two-fold. First, it is to ensure that all women Veterans receive equitable, high quality and comprehensive health care in a sensitive and safe environment at all VA facilities. The care must be of highest quality, and we have been aggressively implementing models of comprehensive care delivery. The goal of a sensitive and safe environment has in some ways been one of our greatest challenges, due in large part to the aging of our infrastructure. We need to look critically at the relationship between creation of a sensitive and safe clinical environment and women Veterans' satisfaction with treatment and perceptions of their care. The second focus in our mission is to be a national leader in the provision of health care for women. That is, the goal is not just having the care of women being as good as the care of men in the VA is, but rather developing a health care system that is a best practice model, demonstrating to the country how to provide good health care to women.

Our office has a long history of collaboration with women's health research investigators. In fact, we frequently engage VA researchers to get their input on ways research can inform VA policy and practice. For example, our program has been working with Dr. Susan Frayne and her research team at VA Palo Alto for the last several years on the development of the Women's Health Evaluation Initiative (WHEI). WHEI has yielded a series of systematically analyzed VA Sourcebooks describing women Veterans' sociodemographic characteristics, health care needs and patterns of care used by my office for policy and planning. ${ }^{12}$, Also funded by our office was the 2008-2009 National Survey of Women Veterans (NSWV), led by Dr. Donna Washington and her team at VA Greater Los Angeles. NSWV represents the first time VA has fielded such a survey in over 20 years. $^{3-5}$ The new VA HSR\&D-funded Women's Health CREATE (http://www.hsrd.research.va.gov/ create/womens_health.cfm ), led by Dr. Elizabeth Yano at VA
Greater Los Angeles and a team of women's health investigators across the country, reflects several years of support and collaboration by WHS and addresses one of our program's top strategic priorities, implementing comprehensive women's health care throughout the VA.

The following outline briefly identifies WHS goals and priorities in key areas for women's health and links these clinical areas to research needs. First, more research is needed focusing on women Veterans' reproductive health and maternity care. In response to the increasing number of women Veterans using the VA for obstetric care, we are developing policies; for example, a maternity health care and coordination program (VHA Handbook 1330.03), which requires a designated maternity care coordinator in each VA facility. The Caregiver Law (PL 11-163) also provided programming for newborn care and piloting childcare, and these programs will need to be evaluated over the next few years. Some VA-supported research has begun to examine pregnancy outcomes, including unplanned pregnancies and preconception behaviors. ${ }^{6-9}$ There has also been a shift in the VA to recognize lesbian, bisexual, and transgender women Veterans and to give them respectful and appropriate medical care. $.^{10},{ }^{11}$

Another WHS priority area is emergency room care. We need to better understand patterns of use of VA and non-VA emergency care, so we initiated a national survey examining capacity and gaps in our emergency departments, and we are launching a large project utilizing virtual simulation for staff education in VA emergency departments. ${ }^{12}$ Given that about $20 \%$ of women Veterans in our primary care clinics use the emergency department every year, there is much to learn about why and how they use emergency department services.

I have often spoken about the fact we need to address VA culture. My overarching goal has been to enhance the language, the practice and the culture of VA to be more inclusive of women Veterans. It is our collective job to give a woman Veteran the best care anywhere. One cultural change we are seeking to bring about is to dispel the notion that in the VA, it is the job of the Women Veteran Program Manager alone or the women Veterans program alone to take care of women. This approach is not applied to any other minority group, but for women Veterans, we have relegated the job of taking care of women to a very small part of the infrastructure. With $15 \%$ of active duty being female, and the corresponding growth in the female Veteran 
population, we must continue to influence the culture so that VA employees realize that it's everyone's job to care for women. One goal is to measure the outcomes of staff education both in terms of gender sensitivity and clinical competency in key women's health areas.

We often ask, "How can the VA be creative in attracting more female Veterans to receive services?" There are public service announcements that deliver messages to the community that women are Veterans and that the VA provides good care, yet women Veterans continue to underutilize VA healthcare. Market penetration has grown from $11 \%$ to $15 \%$ in the last four years, but this is insufficient progress - the market penetration for male Veterans is $22 \%$. The phrase, "if you build it, they will come" comes to mind, but the lesson is not merely building a care infrastructure, but that in building the right kind of healthcare for women locally, it will grow very quickly at that site. Women also tend to tell other women about their healthcare experiences. We have learned that if a site develops a good women's health program then the utilization numbers go up exponentially. Many of the new VA patients have insurance, and yet they affirm that they will continue to use the VA because the care is very good, and recent data show a pattern of retention over 5 years for a cohort of women Veterans who were recently deployed. ${ }^{5}$ When the care is good and the Veteran experiences respected, women Veterans choose VA over other options.

There are a number of strategies we have implemented to help make hospital directors and chiefs of services understand the high priority VA now places on women's health - there are both carrots and sticks. We frequently visit the field to speak with facility and Veterans Integrated Service Network (VISN) Directors. For the first time ever, we have been able to implement "a stick," which is that the VISN performance requirements include reports on progress to reduce gender disparities in preventive care. Six measures with existing gender disparities were selected, and each VISN chose one to address: ischemic heart disease LDL < 100, Diabetics LDL $<100, \mathrm{HbA1C}>9$ or not done, Influenza vaccine age 50-64, Influenza vaccine $>65$, or communication about medication. Each VISN had to identify the existing gender disparity and then specify the target that would be used to gauge success. VISNs had to develop a process for performance improvement and then demonstrate an implementation process to show measurable improvement and narrowing of the gender gap. If VISNs were unable to show measurable improvement, they had to provide a clear explanation for failure to meet their goal. This process led to significant clinical improvements from fiscal year (FY) 2008 to FY 2011 in the identified performance areas. This example demonstrates the value of research/clinical/policy partnerships in accelerating evidence-based implementation and impact. Overall, we have narrowed the gap in gender disparity for prevention on all measures, and eliminated gaps on most indicators.
We are also developing, implementing and influencing VA education initiatives across the board, particularly in terms of delivery of women's health care. Our strategic goal focuses on increasing our understanding of the effects of military service on women's lives. This is an especially opportune area given that more recent women Veterans have different military experience and exposures than many of their counterparts in the past. We have many women who are actively engaged in combat areas, and we need to understand the effects of this service, both positive and negative effects that have been created because of their military service. There are, of course, research efforts in the areas of combat and trauma exposure and many different kinds of mental health conditions. ${ }^{13}$ We partner with Dr. Susan McCutcheon, the Director of Family Services, Women's Mental Health and Military Sexual Trauma for the Office of Mental Health Services in the Department of Veterans Affairs. In the past, $60-70 \%$ of the research conducted for women Veterans has been focused on mental health issues, and it continues to be a high priority.

Implementing comprehensive primary care means offering complete primary care from one designated women's health care provider at one site. It must include PACT (Patient Aligned Care Team-VA's patient centered medical home model), and we are measuring this model with our women's health primary care evaluation tools. There are ongoing prospective and retrospective measures of implementation of primary care for women. To date, we do not know if patients are more satisfied or if clinical care outcomes improve with implementation of this type of systematic care for women, but VA research on these issues will soon be underway.

We also have put tremendous effort into influencing VA education initiatives that were tied to solving how to recruit new providers or retrain existing providers interested in women's health, and then helping to make them proficient. With programs such as the National VA Women's Health Mini-Residency Program, we have now trained 1,500 primary care providers. Another partnership is the Simulation Learning, Education and Research Network (SimLEARN), the VHA's program for simulation in healthcare training (http://www.simlearn.va. gov/). ${ }^{14}$ We also have long supported the advanced fellowships in women Veterans' health and are expanding these fellowships beyond medical fellowships to the allied health groups. ${ }^{15}$ However, research is lacking regarding the clinical outcomes of training providers in women's health beyond self assessment of learning. For example, it would be important to examine changes in provider behavior after receiving training in contraception and teratogenicity of psychotropic medications.

Finally, because Veterans have life-long health care eligibility, we are focusing on the growing proportion of aging women Veteran VA users. Research is needed on chronic conditions, and there is an emerging need for geriatric services. A key priority is to understand more about conditions with high morbidity and mortality for women. For example, we are 
examining how cardiac disease manifests itself in women Veterans and also testing treatment of heart disease provided to women in the VA. Lung cancer is the next big mortality concern for women in the country and VA has very little research on smoking cessation by gender and whether VA can provide specific types of smoking cessation treatments that may be particularly successful for women Veterans. Last but not least, we need to reduce barriers to early cancer screening for women Veterans, including engaging Veterans as partners in their health.

The future for women Veterans' health and health care can be bright, but it will take concerted efforts of VA clinicians, researchers and policy makers, as well as cultural changes within the VA system to get us there. The goals for the VA are to have research inform evidence-based practice and to continue to serve as a national leader for all women's health.

Disclaimer: The views expressed in this editorial are those of the author and do not necessarily reflect the position or policy of the Department of Veterans Affairs or the United States government.

Conflict of interest: The authors declare that they do not have a conflict of interest.

Corresponding Author: Patricia M. Hayes, PhD; Women's Health Services, Patient Care Services, Department of Veterans Affairs, VA Central Office, 1717 H Street, NW, Washington, DC 20420, USA (e-mail: patricia.hayes@va.gov).

\section{REFERENCES}

1. Frayne SM, Phibbs CS, Friedman SA, Berg E, Ananth L, Iqbal S, Hayes PM, Herrera L. Sourcebook: Women Veterans in the Veterans Health Administration. Volume 1. Sociodemographic Characteristics and Use of VHA Care. Women's Health Evaluation Initiative, Women Veterans Health Strategic Health Care Group, Veterans Health Administration, Department of Veterans Affairs, Washington DC. December 2010.
2. Frayne SM, Phibbs CS, Friedman SA, Saechao F, Berg E, Balasubramanian $\mathrm{V}$, Bi $\mathbf{X}$, Iqbal $\mathrm{S}$, Mattocks $\mathrm{K}$, Haskell $\mathrm{S}$, Zephyrin L, Torgal A, Whitehead A, Hayes PM. Sourcebook: Women Veterans in the Veterans Health Administration. Volume 2. Sociodemographics and Use of VHA and Non-VA Care (Fee). Women's Health Evaluation Initiative, Women's Health Services, Veterans Health Administration, Department of Veterans Affairs, Washington DC. October 2012.

3. Washington DL, Davis TD, Der-Martirosian C, Yano EM. PTSD risk and mental health care engagement in a multi-war era community sample of women veterans. J Gen Intern Med. 2013. doi:10.1007/ s11606-012-2303-2 [Epub ahead of print].

4. Washington DL, Bean-Mayberry B, Riopelle D, Yano EM. Access to care for women veterans: delayed healthcare and unmet need. J Gen Intern Med. 2011;26(Suppl 2):655-61.

5. Hamilton AB, Frayne SM, Cordasco KM, Washington DL. Factors related to attrition from VA healthcare use: findings from the national survey of women veterans. J Gen Intern Med. 2013. doi:10.1007/ s11606-013-2347-y.

6. Goyal V, Borrero S, Schwarz EB. Unintended pregnancy and contraception among active-duty. Am J Obstet Gynecol. 2012;206(6):463-9.

7. Borrero S, Mor MK, Zhao X, McNeil M, Ibrahim S, Hayes $\mathbf{P}$. Contraceptive care in the VA health care system. Contraception. 2012;85(6):580-8.

8. Mattocks KM, Nikolajski C, Haskell S, Brandt C, McCall-Hosenfeld J, Yano E, Pham T, Borrero S. Women veterans' reproductive health preferences and experiences: a focus group analysis. Womens Health Issues. 2011;21(2):124-9.

9. Schwarz EB, Mattocks K, Brandt C, Borrero S, Zephyrin LC, Bathulapalli H, Haskell S. Counseling of female Veterans about risks of medication-induced birth defects. J Gen Intern Med. 2013. doi: 10.1007/s11606-012-2240-0.

10. Levahot K, Simpson TL. Incorporating lesbian and bisexual women into women veterans' health priorities. J Gen Intern Med. 2013. doi:10.1007/ s11606-012-2291-2.

11. Mattocks KM, Sadler S, Yano EM, Krebs EE, Zephyrin L, Brandt C, Kimerling R, Sandfort T, Dichter ME, Weiss JJ, Allison J, Haskell S. Sexual victimization, health status, and VA healthcare utilization among lesbian and bisexual OEF/OIF veterans. J Gen Intern Med. 2013. doi:10.1007/s11606-013-2357-9.

12. Cordasco KM, Zephyrin LC, Kessler CS, Mallard M, Canelo I, Rubenstein LV, Yano EM. An inventory of VHA emergency departments' resources and processes for caring for women. J Gen Intern Med. 2013. doi:10.1007/s11606-012-2327-7.

13. Mattocks KM, Haskell SG, Krebs EE, Justice AC, Yano EM, Brandt C. Women at war: understanding how women veterans cope with combat and military sexual trauma. Soc Sci Med. 2012;74(4):537-45.

14. Bryan T, Snyder E. The clinical breast exam: a skill that should not be abandoned. J Gen Intern Med. 2013. doi:10.1007/s11606-013-2373-9 [Epub ahead of print].

15. Tilstra SA, Kraemer KL, Rubio DM, McNeil MA. VA fellowships: a successful model for developing female leaders in women's health and academic medicine. J Gen Intern Med. 2013. doi:10.1007/s11606-0122306-z [Epub ahead of print]. 